\section{Estrategia Regional de Implementación para América Latina y el Caribe del Plan de Acción Internacional de Madrid sobre el Envejecimiento ${ }^{1}$}

Palabras clave: envejecimiento de la población, envejecimiento, anciano, servicios de salud para ancianos, América Latina, región del Caribe.

Adoptada por los países de América Latina y el Caribe que participaron en la Conferencia Regional Intergubernamental sobre Envejecimiento, celebrada en Santiago, Chile, del 19 al 21 de noviembre de 2003. El texto completo de la Estrategia Regional se presenta en http://www.cepal.org/celade/santiago2003
En América Latina y el Caribe el envejecimiento poblacional, aun con distintos ritmos de avance, es un proceso generalizado; todos los países de la Región marchan hacia sociedades más envejecidas. Consciente de las repercusiones que este fenómeno tiene en el desarrollo de la Región y cumpliendo con el mandato de la Segunda Asamblea Mundial sobre el Envejecimiento, la Comisión Económica para América Latina y el Caribe (CEPAL) apoyó, junto con los organismos del Grupo Interinstitucional sobre Envejecimiento (conformado por la CEPAL, el Fondo de Población de las Naciones Unidas (FNUAP), la Organización Panamericana de la Salud (OPS), la Organización Internacional del Trabajo (OIT), el Banco Interamericano de Desarrollo (BIID), el Banco Mundial y el Programa sobre el Envejecimiento, de las Naciones Unidas), al Gobierno de Chile en la organización de la Conferencia Regional Intergubernamental sobre el Envejecimiento, que se celebró en Santiago, Chile, del 19 al 21 de noviembre de 2003. Esta Conferencia fue la primera reunión de carácter intergubernamental en la materia realizada en la Región y constituyó una instancia de análisis e intercambio respecto de la situación del envejecimiento y las personas mayores. En ella los países de la Región acordaron la presente Estrategia Regional de Implementación para América Latina y el Caribe del Plan de Acción Internacional de Madrid sobre el Envejecimiento.

La Conferencia se originó en la necesidad de dar seguimiento en la Región a la Segunda Asamblea Mundial sobre el Envejecimiento, que se celebró en Madrid en abril de 2002, marcando un hito significativo para el mundo y la Región. Actualmente, gracias a los acuerdos alcanzados en Madrid, se ha reconocido que el envejecimiento poblacional es un fenómeno mundial y que, más allá de las cifras, implica una profunda transformación de nuestras sociedades que exige una readecuación de políticas y programas. En dicha Asamblea se adoptaron dos documentos oficiales: la Declaración Política y el Plan de Acción Internacional de Madrid sobre el Envejecimiento. En el primer documento se establecen los compromisos de los gobiernos para responder a los desafíos que plantea el envejecimiento a las formas de organización social, económica y cultural, mientras que el segundo constituye un instrumento programático en el que se proponen más de un centenar de recomendacio- 
nes en tres áreas prioritarias: 1) las personas de edad y el desarrollo; 2) el fomento de la salud y el bienestar en la vejez, y 3) la creación de un entorno propicio y favorable. El Plan de Acción, como ha señalado el propio Secretario General de las Naciones Unidas, es un nuevo y ambicioso programa para encarar el reto del envejecimiento en el siglo XXI.

En la Estrategia Regional se plantean metas, objetivos y recomendaciones para la acción en favor de las personas mayores en cada una de las tres áreas prioritarias acordadas en Madrid. Representa un marco de referencia regional que los países deben adaptar a sus realidades nacionales con el fin de responder eficazmente a las necesidades e intereses de las personas de edad avanzada, propiciando la creación de condiciones que favorezcan un envejecimiento individual y colectivo con seguridad y dignidad. El desafío actual está centrado en la implementación de dichos acuerdos, para lo cual los países de la Región deben hacer suyo este instrumento y diseñar creativamente las medidas que contribuyan a su concreción.

En la Estrategia Regional se reconocen los desafíos derivados del proceso de envejecimiento poblacional y se examinan las oportunidades que surgen, entre las cuales figuran la construcción de sociedades más democráticas y solidarias desde el punto de vista de la edad, el ejercicio de los derechos y responsabilidades en la vejez y la constitución de sujetos sociales reconocidos como una "nueva fuerza para el desarrollo".

Las metas generales y objetivos específicos de cada una de las áreas prioritarias, así como los mecanismos de seguimiento y evaluación de la Estrategia Regional, se presentan a continuación.

\section{Área prioritaria: las personas de edad y el desarrollo}

Meta general. Proteger los derechos humanos de las personas mayores y crear las condiciones de seguridad económica, de participación social y de educación que propicien la satisfacción de las necesidades básicas de ese grupo de edad y su plena inclusión en la sociedad y el desarrollo.

Objetivos.

1. Promover los derechos humanos de las personas mayores.

2. Facilitar el acceso, en condiciones de igualdad, al empleo decoroso, a la formación continua y al crédito para empresas propias o comunitarias.

3. Promover y facilitar la inclusión laboral formal de los adultos mayores.
4. Ampliar y mejorar la cobertura de pensiones, tanto contributivas como no contributivas.

5. Crear las condiciones adecuadas para articular la plena participación de las personas mayores en la sociedad, con el fin de favorecer su habilitación como grupo social y fortalecer el ejercicio de una ciudadanía activa.

6. Promover la igualdad de oportunidades y el acceso a la educación a lo largo de toda la vida.

\section{Área prioritaria: el fomento de la salud y el bienestar en la vejez}

Meta general. Darles acceso a las personas mayores a servicios de salud integrales y adecuados a sus necesidades, que garanticen una mejor calidad de vida en la vejez y el mantenimiento de su funcionalidad y autonomía.

\section{Objetivos.}

1. Promover la cobertura universal de las personas mayores por los servicios de salud, incorporando el envejecimiento como componente esencial de las leyes y políticas nacionales en torno a la salud.

2. Establecer un servicio integral de salud que responda a las necesidades de las personas adultas mayores, fortaleciendo y reorientando para ello los servicios existentes y creando aquellos que fueran necesarios.

3. Fomentar conductas personales y ambientes saludables mediante leyes, políticas, programas y acciones en los ámbitos nacional y comunitario.

4. Crear marcos legales y mecanismos adecuados para proteger los derechos de las personas mayores que utilizan los servicios de atención prolongada.

5. Promover la formación de recursos humanos mediante el diseño y la implementación de un plan nacional de capacitación en gerontología y geriatría para los prestadores actuales y futuros de servicios de salud, en todos los niveles de atención, subrayando el nivel de la atención primaria.

6. Elaborar y utilizar instrumentos para mejorar el conocimiento del estado de salud de las personas mayores y vigilar sus cambios.

\section{Área prioritaria: creación de un entorno propicio y favorable}

Meta general. Lograr que las personas mayores gocen de entornos físicos, sociales y culturales que 
potencien su desarrollo y favorezcan el ejercicio de derechos y deberes en la vejez.

Objetivos.

1. Adaptar el entorno físico a las características y necesidades de las personas mayores para lograr una vida independiente en la vejez.

2. Mejorar la disponibilidad, sostenibilidad y adecuación de los sistemas de apoyo social para personas mayores.

3. Eliminar todas las formas de discriminación y maltrato en contra de las personas mayores.

4. Fomentar una imagen positiva de la vejez.

\section{Aplicación y seguimiento de la Estrategia Regional}

Meta general. Alentar a cada país de la Región a impulsar las acciones necesarias para lograr la plena ejecución de la Estrategia Regional y establecer los mecanismos para su aplicación, seguimiento, evaluación y revisión, de acuerdo con sus propias realidades.

Objetivos.

1. Tener en cuenta el envejecimiento poblacional en todos los ámbitos de la política pública, con el propósito de adecuar las intervenciones del Estado al cambio demográfico y a la construcción de una sociedad para todas las edades.

2. Conseguir asistencia técnica mediante la cooperación entre países y el apoyo de los organismos internacionales para formular políticas y programas sobre el envejecimiento.

3. Elaborar y poner en práctica un sistema de indicadores específicos que sirva de referencia en el seguimiento y evaluación de la situación de las personas mayores en los niveles nacional y regional.

4. Realizar y promover investigaciones sobre los principales aspectos del envejecimiento en los niveles nacional y regional.

5. Solicitar a la CEPAL y a otras organizaciones pertinentes que propicien contactos con todos los países de la Región, haciéndoles llegar el ofrecimiento formal de apoyo del Grupo Interinstitucional para echar a andar los mecanismos necesarios que permitan aplicar adecuadamente los compromisos emanados de esta Conferencia.

\section{Evaluación y revisión}

La Estrategia Regional ofrece un marco para la concreción de las medidas acordadas, que deberán adaptarse a la situación de cada país. En un plazo de seis meses con posterioridad al término de la Conferencia se definirían las metas específicas y los mecanismos para el seguimiento de las políticas y programas nacionales. De acuerdo al documento de la Estrategia, los avances logrados y las metas definidas en el nivel nacional se darían a conocer en la reunión del Comité Especial sobre Población y Desarrollo del período de sesiones de la CEPAL, a celebrarse en el marco del trigésimo período de sesiones en San Juan, Puerto Rico, a fines de junio de 2004. En cumplimiento de este acuerdo, en dicha reunión algunos países de la Región presentaron sus avances en la implementación del Plan de Acción de Madrid.

Para fortalecer este acuerdo y permitir un mejor seguimiento, la CEPAL, junto con los organismos del Grupo Interinstitucional sobre Envejecimiento, programaron reuniones subregionales para evaluar la implementación de la Estrategia Regional. Con este objetivo se celebró del 10 al 12 de noviembre de 2004 en San Salvador, El Salvador, la Reunión de Expertos sobre Envejecimiento-II Foro Centroamericano y del Caribe sobre Políticas para Adultos Mayores, con la participación de las instituciones rectoras en materia de envejecimiento de los países de Centroamérica y el Caribe hispano, México y Panamá, así como expertos de organizaciones internacionales y figuras del mundo académico y de la sociedad civil. Del mismo modo, el 8 de noviembre de 2004 se celebró en Puerto España, Trinidad y Tabago, el Simposio Caribeño sobre Envejecimiento Poblacional, dirigido a examinar los adelantos observados en los países del Caribe no hispano. Para noviembre de 2005 se tiene previsto celebrar en Buenos Aires, Argentina, un evento similar para los países de América del Sur.

La CEPAL, siguiendo con lo establecido en la Estrategia y en colaboración con los organismos del Grupo Interinstitucional sobre Envejecimiento, continuará actuando como Secretaría Técnica y realizando actividades de apoyo a los países para facilitar el cumplimiento de los acuerdos adoptados en la Conferencia. 